\title{
Conversão de Glicerol a Ácido lático em meio alcalino utilizando catalisadores de cobre suportado em $\mathrm{SiO}_{2}$
}

Conversion of Glycerol to Lactic Acid in an allkaline medium using $\mathrm{SiO}_{2}$ supported copper catalysts Conversión de Glicerol a Ácido Láctico en medio alcalino utilizando catalizadores de cobre soportados en $\mathrm{SiO}_{2}$

Recebido: 12/01/2022 | Revisado: 20/01/2022 | Aceito: 22/01/2022 | Publicado: 24/01/2022

\author{
Andreza de Faria Alves Cruz \\ ORCID: https://orcid.org/0000-0003-1321-3024 \\ Universidade Federal do Rio de Janeiro, Brasil \\ E-mail: andrezafalvesc@gmail.com \\ Germildo Juvenal Muchave \\ ORCID: https://orcid.org/0000-0003-1714-0336 \\ Universidade Federal do Rio de Janeiro, Brasil \\ E-mail: germildomuchave@gmail.com \\ Donato Alexandre Gomes Aranda \\ ORCID: https://orcid.org/0000-0002-5736-4118 \\ Universidade Federal do Rio de Janeiro, Brasil \\ E-mail: donato@eq.ufrj.br
}

\begin{abstract}
Resumo
O ácido lático é um dos materiais de alto valor agregado que pode ser obtido por meio do glicerol, tendo as mais variadas aplicações em diferentes indústrias, com atual destaque por sua importante aplicação na obtenção de poliácido lático (PLA). O objetivo deste trabalho foi a produção de ácido lático a partir de glicerol em meio alcalino utilizando dois diferentes catalisadores de cobre suportados em sílica $\left(\mathrm{SiO}_{2}\right)$ com $5 \%$ de cobre sobre a massa da solução de glicerol, utilizando um sistema de reação em batelada simples. Para todos os catalisadores, os testes catalíticos foram realizados com uma razão mássica de $\mathrm{NaOH} /$ glicerol de 1:1 e uma concentração de $\mathrm{NaOH}$ igual a $0,6 \mathrm{M}$. Com o catalisador $9 \% \mathrm{Cu} / \mathrm{SiO}_{2}-\mathrm{D} 2$, os testes catalíticos foram realizados em tempo de $6 \mathrm{~h}$, utilizando temperaturas de $220^{\circ} \mathrm{C}, 250^{\circ} \mathrm{C}, 280^{\circ} \mathrm{C}$ e $300^{\circ} \mathrm{C}$, com concentrações de glicerol de $0,6 \mathrm{M}$ e $3,3 \mathrm{M}$. Os resultados de conversão de glicerol $(58,9 \%)$ e seletividade ao ácido lático $(69,4 \%)$ foram satisfatórios em temperaturas mais altas $\left(280^{\circ} \mathrm{C}\right.$ e $\left.300^{\circ} \mathrm{C}\right)$. Os testes com o catalisador $9 \% \mathrm{Cu} / \mathrm{SiO}_{2}-\mathrm{F} 1$ foram realizados utilizando a concentração de glicerol igual a $0,6 \mathrm{M}$, tempo de reação de 6 horas e temperaturas de $250^{\circ} \mathrm{C}, 280^{\circ} \mathrm{C}$ e $300^{\circ} \mathrm{C}$. Os resultados indicaram uma boa conversão de glicerol $(54,0 \%)$ e seletividade ao ácido lático $(59,8 \%)$ em temperaturas mais elevadas $\left(300^{\circ} \mathrm{C}\right)$. Assim, concluiu-se que os catalisadores foram favoráveis nestas reações. A temperatura de reação combinada com o tempo de reação mostraram-se parâmetros essenciais na avaliação da conversão e seletividade ao ácido lático.
\end{abstract}

Palavras-chave: Ácido lático; Catalisadores; Cobre; Glicerol.

\begin{abstract}
Lactic acid is one of the materials with high added value that can be obtained through glycerol, having the most varied applications in different industries, with current emphasis on its important application in obtaining polylactic acid (PLA). The objective of this work was the production of lactic acid from glycerol in an alkaline medium using two different copper catalysts supported on silica $\left(\mathrm{SiO}_{2}\right)$ with $5 \%$ copper on the mass of the glycerol solution, using a simple batch reaction system. For all catalysts, the catalytic tests were carried out with a $\mathrm{NaOH} / \mathrm{glycerol}$ mass ratio of 1:1 and a $\mathrm{NaOH}$ concentration equal to $0.6 \mathrm{M}$. With the catalyst $9 \% \mathrm{Cu} / \mathrm{SiO}_{2}-\mathrm{D} 2$, the catalytic tests were carried out in a reaction time of 6 hours, using temperatures of $220^{\circ} \mathrm{C}, 250^{\circ} \mathrm{C}, 280^{\circ} \mathrm{C}$ and $300^{\circ} \mathrm{C}$, with glycerol concentrations of 0.6 $\mathrm{M}$ and 3.3 M. The results of glycerol conversion $(58,9 \%)$ and selectivity of lactic acid $(69,4 \%)$ were satisfactory at higher temperatures $\left(280^{\circ} \mathrm{C}\right.$ and $\left.300^{\circ} \mathrm{C}\right)$. Tests with the catalyst $9 \% \mathrm{Cu} / \mathrm{SiO}_{2}-\mathrm{F} 1$ were carried out using the glycerol concentration equal to $0.6 \mathrm{M}$, reaction time of 6 hours and temperatures of $250^{\circ} \mathrm{C}, 280^{\circ} \mathrm{C}$ and $300^{\circ} \mathrm{C}$. The results indicated a good conversion of glycerol $(54.0 \%)$ and selectivity to lactic acid $(59.8 \%)$ at higher temperatures $\left(300^{\circ} \mathrm{C}\right)$. Thus, it was concluded that the catalysts were favorable in these reactions. The reaction temperature combined with the reaction time proved to be essential parameters in evaluating the conversion and selectivity to lactic acid.
\end{abstract}

Keywords: Lactic acid; Catalysts; Copper; Glycerol.

\section{Resumen}

El ácido láctico es uno de los materiales de alto valor agregado que se puede obtener a través del glicerol, teniendo las más variadas aplicaciones en diferentes industrias, destacándose actualmente su importante aplicación en la obtención 
de ácido poliláctico (PLA).El objetivo de este trabajo fue la producción de ácido láctico a partir de glicerol en medio alcalino utilizando dos catalizadores de cobre diferentes soportados sobre sílice ( $\mathrm{SiO} 2)$ con $5 \%$ de cobre sobre la masa de la solución de glicerol, utilizando un sistema de reacción discontinuo simple. Para todos los catalizadores, las pruebas catalíticas se realizaron con una relación másica de $\mathrm{NaOH} / \mathrm{glicerol} \mathrm{de} \mathrm{1:1} \mathrm{y} \mathrm{una} \mathrm{concentración} \mathrm{de} \mathrm{NaOH}$ igual a 0,6 M. Con el catalizador $9 \% \mathrm{Cu} / \mathrm{SiO} 2-\mathrm{D} 2$, las pruebas catalíticas se realizaron en un tiempo de reacción de 6 horas, utilizando temperaturas de $220^{\circ} \mathrm{C}, 250^{\circ} \mathrm{C}, 280^{\circ} \mathrm{C}$ y $300^{\circ} \mathrm{C}$, con concentraciones de glicerol de $0,6 \mathrm{M}$ y $3,3 \mathrm{M}$. Los resultados de conversión de glicerol $(58,9 \%)$ y selectividad del ácido láctico $(69,4 \%)$ fueron satisfactorios a mayores temperaturas $\left(280^{\circ} \mathrm{C}\right.$ y $\left.300^{\circ} \mathrm{C}\right)$. Las pruebas con el catalizador $9 \% \mathrm{Cu} / \mathrm{SiO} 2-\mathrm{F} 1$ se realizaron utilizando una concentración de glicerol igual a $0,6 \mathrm{M}$, tiempo de reacción de 6 horas y temperaturas de $250^{\circ} \mathrm{C}, 280^{\circ} \mathrm{C}$ y $300^{\circ} \mathrm{C}$. Los resultados indicaron una buena conversión de glicerol $(54,0 \%)$ y selectividad al ácido láctico $(59,8 \%)$ a temperaturas más altas $\left(300^{\circ} \mathrm{C}\right)$. Por lo tanto, se concluyó que los catalizadores fueron favorables en estas reacciones. La temperatura de reacción combinada con el tiempo de reacción resultaron ser parámetros esenciales en la evaluación de la conversión y selectividad al ácido láctico.

Palabras clave: Ácido láctico; Catalizadores; Cobre; Glicerol.

\section{Introdução}

Os desafios decorrentes do uso de fontes fósseis para a produção de combustíveis têm impulsionado a busca por tecnologias que permitam a produção de biocombustíveis, com o objetivo primordial de reduzir o uso de fontes não renováveis (Postaue et al., 2019).

Devido ao expansivo crescimento das emissões de dióxido de carbono $\left(\mathrm{CO}_{2}\right)$ e da alta do consumo de energia, as esferas políticas, econômicas e ambientais passaram a ter como principal objetivo a redução das emissões dos gases de efeito estufa, de forma a ter um meio ambiente mais sustentável, substituindo gradativamente o consumo de energia tradicional pela energia renovável (Shahbaz et al., 2020).

Dentre os combustíveis renováveis, o destaque se dá para a produção de biodiesel, biocombustível normalmente produzido pela transesterificação de óleos vegetais e gorduras animais, gerando glicerol como subproduto. Estima-se que a produção de uma tonelada de biodiesel gere cerca de 110kg de glicerol bruto (Adhikari et al., 2008).

A conversão catalítica do glicerol em ácido lático vem despertando crescente interesse, pois tem múltiplas aplicações em sua forma opticamente pura; como na indústria alimentícia como acidulante, conservante ou inibidor de bactérias deteriorantes; na indústria cosmética e farmacêutica; na produção de outros produtos de maior valor agregado; e na produção de poliácidolático (PLA), um polímero termoplástico biodegradável com ampla aplicabilidade (Maki-Arvela et al., 2014).

A conversão hidrotérmica de glicerol em ácido lático tem sido bastante estudada recentemente como uma rota alternativa ao processo de fermentação comumente utilizado. Este processo apresenta algumas vantagens, como não necessitar de etapas de purificação de derivados do glicerol para a produção do biodiesel (Roy et al., 2011).

A conversão hidrotérmica do glicerol tornou-se um processo bem estudado devido aos resultados promissores obtidos por Kishida et al. (2005) que obtiveram $90 \%$ de rendimento de ácido lático em condições reacionais de $300^{\circ} \mathrm{C}$ e concentrações de glicerol e $\mathrm{NaOH}$ de 0,33 e 1,25 mol L-1, respectivamente; e Ramírez-López et al. (2010), que relataram um rendimento de $84,5 \%$ de ácido lático nas condições reacionais de $2,5 \mathrm{~mol} \mathrm{~L}^{-1}$ de glicerol a $280^{\circ} \mathrm{C}$ por $90 \mathrm{~min}$ (Yin et al., 2017).

O objetivo deste trabalho é produzir ácido lático a partir do glicerol em meio alcalino utilizando dois catalisadores de cobre suportados em sílica $\left(\mathrm{SiO}_{2}\right)$, empregando um sistema de reação em batelada simples, variando parâmetros como temperatura e concentração dos reagentes, a fim de investigar a conversão e a seletividade do produto desejado.

\section{Metodologia}

Segundo Marconi e Lakatos (2017), o presente trabalho se enquadra em uma pesquisa laboratorial. 


\subsection{Materiais}

Hidróxido de sódio (97,0\%) da Vetec; Ácido sulfúrico da Merck; Glicerol (99,5\%); e Acetonitrila (99,9\%) da Sigma Aldrich. Os padrões para análise por HPLC foram lactato de sódio (98,0\%), Piruvaldeído (sol. 40\%), todos obtidos da Sigma Aldrich; Ácido Lático (85,0-90,0\%) obtido da Proquímios; 1,2-propanodiol da Merck.

\subsection{Redução dos catalisadores}

Os dois catalisadores de cobre suportados em sílica $\left(\mathrm{Cu} / \mathrm{SiO}_{2}\right)$ foram sintetizados pela Unidade Protótipo de Catalisadores (PROCAT/UFRJ), prontos para serem utilizados nas reações de conversão hidrotérmica de glicerol a ácido lático. Os catalisadores foram preparados com sílicas de diferentes propriedades texturais, através da metodologia de impregnação úmida, seguindo procedimento similar ao de Huang; Cui; Xue; Zuo; Chen \& Xia (2012).

Antes da utilização na reação de conversão do glicerol, os catalisadores foram submetidos à redução em forno de fabricação própria (LIPCAT/UFRJ), a uma taxa de $1,5^{\circ} \mathrm{C} / \mathrm{h}$, sob fluxo de gás hidrogênio $\left(\mathrm{H}_{2}\right)$ a temperatura de $400^{\circ} \mathrm{C}$, permanecendo nesta temperatura por 4 horas. Depois do tempo de estabilização, ocorreu a redução da temperatura até $30^{\circ} \mathrm{C}$. Após esta etapa, o catalisador foi submetido à continuação da redução em um reator série (Parr Instrument Company, Ilinois, EUA) de $100 \mathrm{~mL}$ a temperatura de $350^{\circ} \mathrm{C}$ em tempo reacional de 15 horas. Para cada redução, foram utilizados $5 \%$ de catalisador $(0,75 \mathrm{~g})$ em relação à massa de solução de glicerol $(15 \mathrm{~g})$.

\subsection{Caracterização dos catalisadores}

\subsubsection{Análise Textural}

As propriedades de textura dos catalisadores foram determinadas empregando-se a fissorção de nitrogênio $\left(\mathrm{N}_{2}\right)$ em equipamento TriStar 3000. O método BET obteve a área específica, o volume específico dos poros e o diâmetro médio dos poros pelo método BJH através de uma isoterma de dessorção. Após a pesagem, as amostras foram submetidas à secagem a $300^{\circ} \mathrm{C}$ sob vácuo por 24 horas. Posteriormente, foram novamente analisados a uma temperatura de $-196^{\circ} \mathrm{C}$, obtendo-se isotermas de adsorção e dessorção de $\mathrm{N}_{2}$, considerando a região de pressão relativa do gás (P/Po) entre 0 a 1.

\subsubsection{Difração de Raios- $X$}

As medidas foram realizadas em difratômetro da marca Rigaku modelo Miniflex II com radiação de CuK $\alpha(30 \mathrm{kV}$ e $15 \mathrm{~mA}$ ), em intervalo de análise de $5 \leq 2 \theta \leq 90^{\circ}$ com passo de $0,05^{\circ}$ e tempo de contagem de 2 segundos por passo. Utilizou-se o método do pó, com amostra pulverizada, fixada em porta amostra por prensagem e submetida a um feixe de raios X. A identificação das fases cristalinas foi realizada por comparação dos difratogramas com padrões difratométricos da base de dados do ICDD (International Center for Diffraction Data, antigo JCPDS - Joint Committee of Powder Diffraction Standards).

\subsubsection{Fluorescência de Raios- $X$}

Para realização desta técnica, foi utilizado um espectrofotômetro da marca Rigaku, modelo Primini, dotado de tubo gerador de raios $\mathrm{X}$ de paládio. As amostras a serem analisadas estavam calcinadas e foram alojadas em porta amostras com o auxílio de filme de propileno de $6 \mu \mathrm{m}$ de espessura.

\subsubsection{Redução à Temperatura Programada de Hidrogênio (TPR-H}

O perfil de redução dos catalisadores foi obtido por análise de redução à temperatura programada (TPR). Esta análise foi realizada em equipamento equipado com detector de condutividade térmica (TCD). Cada catalisador foi pesado para ter $10 \mathrm{mg}$ de fase ativa. Todas as amostras foram pré-tratadas por 30 minutos a $150^{\circ} \mathrm{C}$ sob fluxo de argônio (Ar) (30mL min ${ }^{-1}$ ). 
Após estabilização do sinal do TCD, as amostras foram aquecidas da temperatura ambiente até $1000^{\circ} \mathrm{C}$ com taxa de aquecimento de $10^{\circ} \mathrm{C} \min ^{-1}$ e vazão de $30 \mathrm{~mL} \mathrm{~min}^{-1}$ de $1,8 \% \mathrm{H}_{2} / \mathrm{Ar}$.

\subsubsection{Dessorção de Temperatura Programada de Amônia (TPD-NH3)}

As amostras de catalisadores foram previamente reduzidas in situ utilizando mistura redutora de 1,8\% $\mathrm{H}_{2} / \mathrm{Ar}(30 \mathrm{~mL}$ $\left.\min ^{-1}\right)$ e aquecimento a $800^{\circ} \mathrm{C}\left(10^{\circ} \mathrm{C} \mathrm{min}^{-1}\right)$ com isoterma de 30 minutos. Posteriormente, as amostras foram resfriadas até temperatura ambiente utilizando hélio puro $(\mathrm{He})$ e a adsorção de amônia foi conduzida a $70^{\circ} \mathrm{C}$ com mistura de $\mathrm{NH}_{3} / \mathrm{He}$ com vazão de $30 \mathrm{~mL} \mathrm{~min}^{-1}$ por 30 minutos, seguido de purga com He puro por 60 minutos. A dessorção da amônia quimissorvida foi realizada por aquecimento das amostras até temperatura de $800^{\circ} \mathrm{C}$ em taxa de $20^{\circ} \mathrm{C} \mathrm{min}^{-1}$. A razão $\mathrm{m} / \mathrm{z}=15$ foi utilizada para quantificação da amônia, realizada por integração da área sob a curva intensidade versus Tempo.

\subsection{Testes Catalíticos}

Os testes catalíticos foram realizados em um microrreator série 4590 de $100 \mathrm{~mL}$ (Parr Instrument Company, Illinois, EUA). Catalisadores de cobre suportados em sílica $\left(9 \% \mathrm{Cu} / \mathrm{SiO}_{2}-\mathrm{D}_{2}\right.$ e $\left.9 \% \mathrm{Cu} / \mathrm{SiO}_{2}-\mathrm{F} 1\right)$ foram escolhidos para serem utilizados nestas reações, sendo que os mesmos se diferenciam pela fase utilizada para impregnação do metal ao suporte. As condições de operação foram moduladas por um controlador de reator 4848 (Parr Instrument Company), e os reagentes foram alimentados no reator na razão de massa desejada de glicerol: $\mathrm{NaOH}$ (1:1). Em seguida, o conteúdo do reator foi agitado a 1000 rpm a uma pressão de vácuo de 800psi.

O catalisador inicialmente testado nas reações de conversão foi $9 \% \mathrm{Cu} / \mathrm{SiO}_{2}-\mathrm{D} 2$, com temperaturas entre 220,250 e $280^{\circ} \mathrm{C}$, concentrações de glicerol entre $0,6,3,3$ e $6,6 \mathrm{M}$, com tempo de reação de $6 \mathrm{~h}$ e concentração de $\mathrm{NaOH}$ de $0,6 \mathrm{M}$. O objetivo inicial foi observar a influência dos parâmetros de concentração de glicerol e temperatura de reação. Em seguida, o catalisador testado nas reações de conversão foi $9 \% \mathrm{Cu} / \mathrm{SiO}_{2}-\mathrm{F} 1$, com temperaturas entre 250,280 e $300^{\circ} \mathrm{C}$, concentrações de glicerol e $\mathrm{NaOH}$ de 0,6 M e tempo de reação de 6h. Nesta etapa, o objetivo foi observar a influência dos parâmetros de concentração de glicerol e temperatura de reação, conforme Tabela 1.

Tabela 1: Condições experimentais de reações de conversão do glicerol a ácido lático com o 9\% $\mathrm{Cu} / \mathrm{SiO}_{2}-\mathrm{D} 2$.

\begin{tabular}{cccc}
\hline Reaction & [glic] $(\mathbf{M})$ & $\mathbf{t}(\mathbf{h})$ & $\mathbf{T}\left(\mathbf{~}^{\mathbf{O}} \mathbf{C}\right)$ \\
\hline 1 & 3,3 & 6 & 250 \\
2 & 0,6 & 6 & 250 \\
3 & 3,3 & 6 & 280 \\
4 & 0,6 & 6 & 280 \\
5 & 3,3 & 6 & 220 \\
6 & 0,6 & 6 & 220 \\
7 & 6,6 & 6 & 280 \\
8 & 3,3 & 6 & 290 \\
9 & 0,6 & 6 & 300 \\
\hline
\end{tabular}

Fonte: Autores.

As análises dos produtos das reações foram analisadas por cromatografia líquida de alta performance (HPLC High-perfomance Liquid Chromatography) com equipamento da marca Shimadzu (Quioto, Japão), modelo UHPLC Nexera ${ }^{\mathrm{TM}}$ Series, composto por uma bomba binária, modelo LC-40B XR, um detector por índice de refração, modelo RID-20A e um injetor automático, modelo SIL-40XR. Foi utilizada uma coluna Hi-Plex H (Agilent Technologies, Califórnia, EUA), de 300mm de comprimento por 7,7 $\mathrm{mm}$ de diâmetro interno e $8 \mu \mathrm{m}$ de tamanho de partícula para separar os produtos. A fase móvel utilizada foi $\mathrm{H}_{2} \mathrm{SO}_{4} 0,001 \mathrm{M}+10 \% \mathrm{ACN}$, submetida a um banho de ultrassom por 15 min antes da utilização, segundo método desenvolvido especialmente para estas reações. As análises foram realizadas a uma temperatura constante de $60^{\circ} \mathrm{C}$ 
(temperatura do forno de coluna), com vazão de $0,6 \mathrm{~mL} \mathrm{~min}^{-1}$ e detecção por índice de refração a $60^{\circ} \mathrm{C}$. O tempo total de corrida foi de 20 minutos. Em todas as reações, antes de serem analisadas, as amostras extraídas passaram por filtração com auxílio de filtros descartáveis de $0,20 \mu \mathrm{m}$ de poro. As amostras analisadas por HPLC foram diluídas a 2x, utilizando água ultrapura (Mili-Q) como diluente e injetadas em triplicata, a um volume de injeção de 20,0 $\mu \mathrm{L}$. Os perfis cromatográficos foram analisados e integrados pelo programa LabSolutions (Shimadzu). Curvas de calibração foram feitas para o glicerol, ácido lático, 1,2- propanodiol, acetol, piruvaldeído e lactato de sódio, com a finalidade de quantificar os produtos formados e o glicerol não convertido.

O desempenho dos catalisadores foi avaliado através da conversão do glicerol e da seletividade do ácido lático, de acordo com as equações:

- Conversão de Glicerol (Conv. (\%):

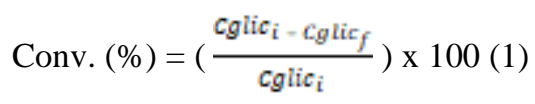

Cglic $c_{i}$ é a concentração molar de glicerol na alimentação, e Cglic $c_{f}$ é a concentração molar de glicerol após a reação.

- Seletividade a Ácido Lático (Sel. (\%)):

$$
\text { Sel. }(\%)=\left(\frac{c_{p}}{\operatorname{cg}_{\mathrm{gli}} \varepsilon_{\mathrm{i}}-\mathrm{cglil}_{f}}\right) \times 100(2)
$$

$\mathrm{C}_{\mathrm{p}}$ é a concentração dos produtos formados, Cgli $c_{\mathfrak{f}}$ é a concentração molar de glicerol na alimentação, e Cglic $c_{f}$ é a concentração molar de glicerol após a reação.

Em relação ao catalisador $\mathrm{Cu} / \mathrm{SiO}_{2}-\mathrm{D} 2$, para avaliação da concentração de glicerol, realizou-se o cálculo do rendimento, conforme (3).

$$
\text { Rendimento }(\%)=\text { Seletividade }(\%) \times \text { Conversão (3) }
$$

\section{Resultados e Discussão}

\subsection{Caracterização dos catalisadores}

\subsubsection{Análises e Propriedades Texturais}

Os resultados obtidos através da análise de FRX dos catalisadores indicam a porcentagem em massa dos óxidos dos componentes metálicos dos catalisadores, com a presença de componentes já previstos na composição dos mesmos (sendo eles $\mathrm{Cu}, \mathrm{Mg}$ e Si). Como esta análise fornece resultados associados à composição sólida da superfície (poros excluídos), as diferenças apresentadas entre as quantidades teoricamente previstas e as quantidades reais obtidas na FRX são aceitáveis. Eles podem ser atribuídos a algum erro experimental durante a preparação dos catalisadores, como visto na Tabela 2.

Tabela 2: Composição química (\% mássica) dos catalisadores de cobre.

\begin{tabular}{ccccc}
\hline Catalisadores & \multicolumn{2}{c}{$\mathrm{Cu} / \mathrm{SiO}_{2}-\mathrm{D2}$} & \multicolumn{2}{c}{$\mathrm{Cu} / \mathrm{SiO}_{2}-\mathrm{F1}$} \\
\hline \multirow{2}{*}{ Composição (\%) } & $\mathrm{SiO}_{2}$ & $\mathbf{C u O}$ & $\mathrm{SiO}_{2}$ & $\mathbf{C u O}$ \\
\hline
\end{tabular}

Fonte: Autores.

Os resultados das análises de DRX para os catalisadores de cobre podem ser visualizados na Figura 1. Observa-se que todos os catalisadores possuem perfil semelhante. No entanto, há uma diferença na intensidade dos picos. Os picos de 
difração $2 \theta=35,38,48$ e $68^{\circ}$ são atribuídos ao $\mathrm{CuO}$, correspondendo a um sistema cristalino monoclínico. O pico de difração em $2 \theta=22^{\circ}$ é característico do óxido de silício $\left(\mathrm{SiO}_{2}\right)$. Os picos mais intensos para $\mathrm{CuO}$ ocorrem em $2 \theta=35^{\circ}$ e $2 \theta=38^{\circ}$, o que corrobora com o descrito por Wang et al. (2013). Pode-se concluir, através dos resultados obtidos, que o grande tamanho do cristal proporciona boa estabilidade e melhor contato entre as partículas de $\mathrm{Cu}$ e $\mathrm{SiO}_{2}$, favorecendo o desempenho do catalisador $\mathrm{Cu} / \mathrm{SiO}_{2}$.

Figura 1: Difratograma de raios- $X$ dos catalisadores.

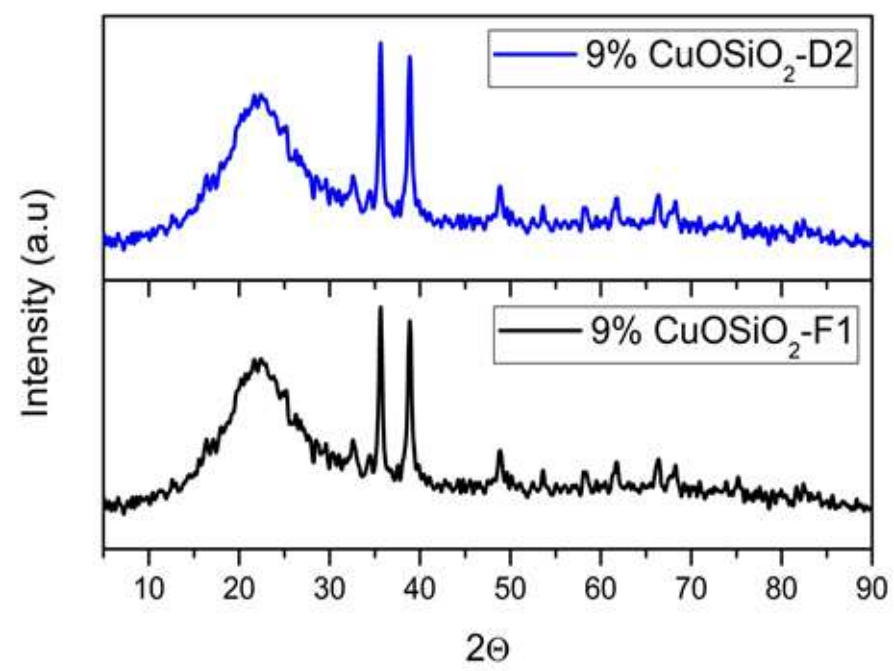

Fonte: Autores.

$\mathrm{O}$ processo de adsorção/dessorção de $\mathrm{N}_{2}$ a $77 \mathrm{~K}$ permitiu a determinação dos principais parâmetros texturais dos catalisadores de $\mathrm{Cu} / \mathrm{SiO}_{2}$, conforme apresentado na Tabela 3 .

Tabela 3: Propriedades texturais dos catalisadores $\left(\mathrm{Cu} / \mathrm{SiO}_{2}\right)$ e suporte $\left(\mathrm{SiO}_{2}\right)$.

\begin{tabular}{|c|c|c|c|}
\hline Catalisadores & $\begin{array}{c}\text { Área específica }\left(\mathrm{m}^{2} \mathrm{~g}^{-}\right. \\
1)\end{array}$ & $\begin{array}{c}\text { Volume de poro }\left(\mathrm{cm}^{3}\right. \\
\left.\mathrm{g}^{-1}\right)\end{array}$ & Diâmetro do poro $\left(\mathrm{A}^{0}\right)$ \\
\hline Suporte $\mathrm{SiO}_{2}$ & 496,0 & 0,50 & - \\
\hline $9 \% \mathrm{CuSiO}_{2}-\mathrm{F} 1$ & 150,5 & 0,51 & 119,2 \\
\hline 9\% $\mathrm{CuSiO}_{2}-\mathrm{D} 2$ & 76,2 & 0,15 & 52,7 \\
\hline
\end{tabular}

Fonte: Autores.

De acordo com a classificação dos diâmetros médios dos poros, observa-se na Tabela 3 que esses valores estão incluídos em uma faixa característica dos mesoporos, segundo a IUPAC (SING, 1985). Ao comparar os valores de área superficial do suporte com os catalisadores, a maior área superficial BET do suporte catalítico poderá promover um aumento na dispersão de $\mathrm{Cu}$. É necessário destacar que a agitação da reação é suficiente para desprezar os efeitos da transferência de massa. É possível observar que o catalisador $9 \% \mathrm{CuSiO}_{2}-\mathrm{F} 1$ apresentou menor redução de área específica $\left(496,0 \mathrm{~cm}^{2} \mathrm{~g}^{-1}\right.$ para $\left.150,5 \mathrm{~cm}^{2} \mathrm{~g}^{-1}\right)$, enquanto que o $9 \% \mathrm{CuSiO}_{2}-\mathrm{D} 2$ apresentou maior redução da área específica $\left(496,0 \mathrm{~cm}^{2} \mathrm{~g}^{-1}\right.$ para $\left.76,2 \mathrm{~cm}^{2} \mathrm{~g}^{-1}\right)$. Esse fato pode estar relacionado com a distribuição de poros na sílica, com deposição e bloqueio pelas partículas metálicas (Tran et al., 2016). É possível observar que, ao comparar os catalisadores com o suporte utilizado, o catalisador $9 \% \mathrm{CuSiO}_{2}-\mathrm{D} 2$ apresentou redução no volume dos poros, que pode ser explicado por deposição de cobre no interior do poro ou bloqueio de poros (hipótese que precisa de outras análises para ser confirmada). Para o catalisador $9 \% \mathrm{CuSiO}_{2}-\mathrm{F} 1$, ocorreu pequeno 
aumento do volume dos poros, mas que não chega a ser significativo, o que indica que não ocorreu quase mudança em relação ao bloqueio de poros nesse catalisador (Huynh et al., 2016).

Figura 2: Isotermas de adsorção-dessorção de $\mathrm{N}_{2}$ dos catalisadores (A) $9 \% \mathrm{Cu} / \mathrm{SiO}_{2}-\mathrm{D} 2$ e (B) $9 \% \mathrm{Cu} / \mathrm{SiO}_{2}-\mathrm{F} 1$.

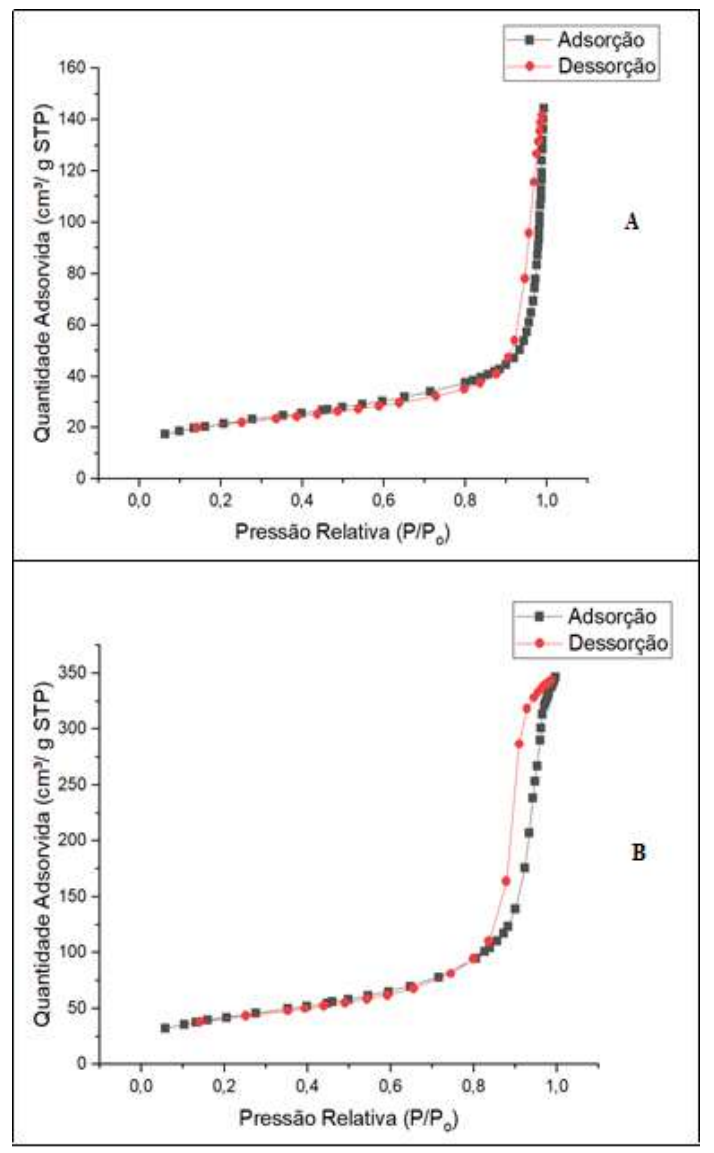

Fonte: Autores.

De acordo com a Figura 2, as isotermas de adsorção-dessorção de $\mathrm{N}_{2}$ dos catalisadores $\mathrm{Cu} / \mathrm{SiO}_{2}-\mathrm{D} 2$ e $\mathrm{Cu} / \mathrm{SiO}_{2}-\mathrm{F} 1$ exibiram um padrão semelhante ao tipo III, sem histerese; características de sistemas em que as moléculas do adsorvato apresentam maior interação entre si do que com o próprio sólido (SING, 1985; WEBB \& ORR, 1997).

\subsubsection{Propriedades Redutivas}

A Figura 3 mostra o perfil de redução de temperatura programada (TPR- $\left.\mathrm{H}_{2}\right)$ para os catalisadores $9 \% \mathrm{Cu} / \mathrm{SiO}_{2}-\mathrm{D} 2 \mathrm{e}$ $9 \% \mathrm{Cu} / \mathrm{SiO}_{2}-\mathrm{F} 1$. Os perfis de TPR- $\mathrm{H}_{2}$ indicaram que a redução de $\mathrm{CuO}$ ocorreu em temperaturas abaixo de $600 \mathrm{~K}$ em todos os catalisadores, sugerindo pequenas partículas de $\mathrm{CuO}$ e alta dispersão de cobre (Flores et al., 2011).

Observa-se que o catalisador $9 \% \mathrm{Cu} / \mathrm{SiO}_{2}$-D2 apresentou picos de redução a $277,7,349,4$ e $411,3{ }^{\circ} \mathrm{C}$, todos acima do intervalo descrito na literatura $\left(200 \mathrm{a} 400^{\circ} \mathrm{C}\right)$ para redução da fase ativa de $\mathrm{CuO}$, o que indica interação mais significativa entre metal $(\mathrm{Cu})$ e suporte catalítico $\left(\mathrm{SiO}_{2}\right)$ (Dussault et al., 2005). Já o catalisador $9 \% \mathrm{Cu} / \mathrm{SiO}_{2}-\mathrm{F} 1$ apresentou três picos de redução, a $326,411 \mathrm{e} 498.4^{\circ} \mathrm{C}$, dentro da faixa descrita na literatura (Dussault et al., 2005). 
Research, Society and Development, v. 11, n. 2, e25011225652, 2022

(CC BY 4.0) | ISSN 2525-3409 | DOI: http://dx.doi.org/10.33448/rsd-v11i2.25652

Figura 3: Perfis de TPR- $\mathrm{H}_{2}$ dos catalisadores (A) $9 \% \mathrm{Cu} / \mathrm{SiO}_{2}-\mathrm{D} 2$ e (B) $9 \% \mathrm{Cu} / \mathrm{SiO}_{2}-\mathrm{F} 1$
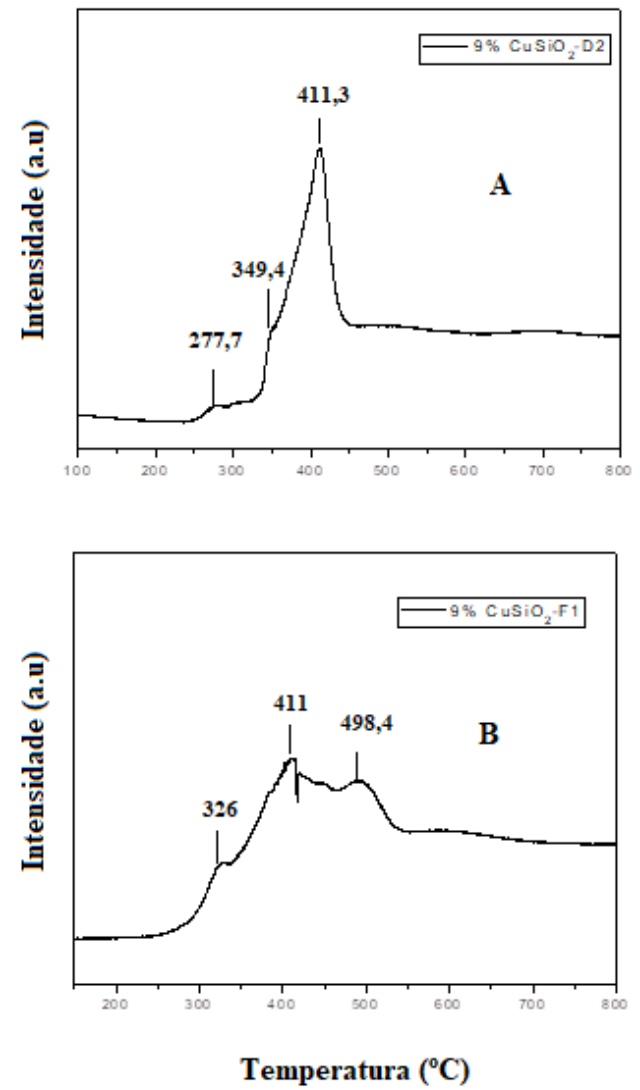

Fonte: Autores.

\subsubsection{Acidez e dispersão de cobre}

A Figura 4 mostra o perfil de dessorção de temperatura programada dos catalisadores $9 \% \mathrm{Cu} / \mathrm{SiO}_{2}-\mathrm{F} 1$ e $9 \% \mathrm{Cu} / \mathrm{SiO}_{2}-$ D2. Pode-se observar que os catalisadores suportados em sílica possuem interações fracas e fortes com a amônia. Ou seja, o catalisador $9 \% \mathrm{Cu} / \mathrm{SiO} 2-\mathrm{F} 1$ possui quatro picos, a $250^{\circ} \mathrm{C}, 300^{\circ} \mathrm{C}, 500^{\circ} \mathrm{C}$ e $625^{\circ} \mathrm{C}$, e o catalisador $9 \% \mathrm{Cu} / \mathrm{SiO}_{2}-\mathrm{D} 2$ tem três picos, a $280^{\circ} \mathrm{C}, 330^{\circ} \mathrm{C}$ e $550^{\circ} \mathrm{C}$. Para o catalisador $9 \% \mathrm{Cu} / \mathrm{SiO}_{2}-\mathrm{F} 1$, há um pico a $625^{\circ} \mathrm{C}$, característico de amônia fortemente adsorvida, segundo a literatura de Liu et al. (2014). 
Figura 4: Perfil de dessorção de TPD- $\mathrm{NH}_{3}$ do catalisador A) $9 \% \mathrm{Cu} / \mathrm{SiO}_{2}-\mathrm{F} 1$ e B) $9 \% \mathrm{Cu} / \mathrm{SiO}_{2}-\mathrm{D} 2$.

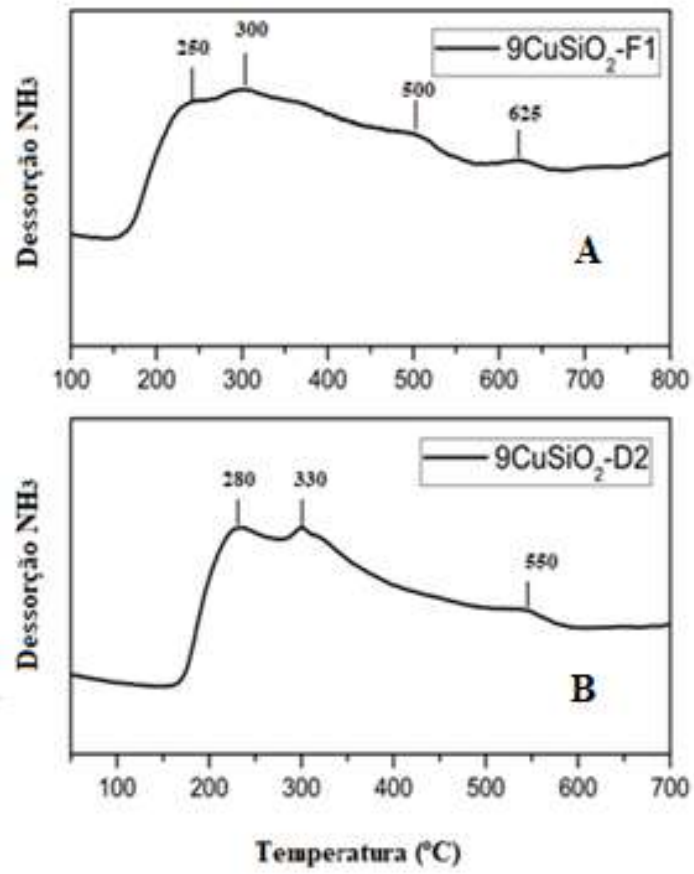

Fonte: Autores.

Tabela 4: Quantidade de amônia adsorvida e razão de sítio ácido forte/fraco.

\begin{tabular}{ccccc}
\hline Catalisador & \multicolumn{2}{c}{$\begin{array}{l}\text { NH3 } \\
\text { 6/gcat) }\end{array}$} & $\left(\right.$ molNH3.1e $^{-}$ & \multicolumn{2}{c}{ Razão de sítios ácidos (\%) } \\
\cline { 3 - 4 } & & Fraco & Forte \\
\hline $9 \% \mathrm{Cu} / \mathrm{SiO}_{2}-\mathrm{D} 2$ & 400,98 & 51,78 & 48,22 \\
$9 \% \mathrm{Cu} / \mathrm{SiO}_{2}-\mathrm{F} 1$ & 614,67 & 36,55 & 63,45 \\
\hline
\end{tabular}

Fonte: Autores.

Conforme se pode observar na Tabela 4, a acidez dos catalisadores foi medida por dessorção termoprogramada de $\mathrm{NH}_{3}\left(\mathrm{TPD}-\mathrm{NH}_{3}\right)$, e o catalisador $9 \% \mathrm{Cu} / \mathrm{SiO}_{2}-\mathrm{F} 1$ apresentou maior quantidade de amônia adsorvida, que pode ser relacionado à sua maior área superficial quando comparado ao outro catalisador, o que corrobora com o descrito por Jiao et al. (2012), além de uma maior razão de sítios ácidos fortes quanto comparado ao catalisador $9 \% \mathrm{Cu} / \mathrm{SiO}_{2}-\mathrm{F} 1$. Isso pode ser explicado em decorrência da diferença de propriedade estrutural de cada catalisador. Além disso, o catalisador $9 \% \mathrm{Cu} / \mathrm{SiO}_{2}-\mathrm{F} 1$ possui maior razão de sítios ácidos fortes quando comparado aos sítios ácidos fracos, o que pode ser explicado em decorrência da presença de maior quantidade de picos em regiões de alta temperatura, que, conforme Karge et al. (1991), indica a dessorção da amônia desses sítios ácidos fortes.

\subsection{Testes Catalíticos}

\subsubsection{Influência da concentração de glicerol}

Para o catalisador $\mathrm{Cu} / \mathrm{SiO}_{2}-\mathrm{D} 2$, foram avaliadas as influências das temperaturas na faixa de 220 a $280^{\circ} \mathrm{C}$, e da concentração de glicerol, utilizando os parâmetros de conversão e seletividade para o ácido lático, a fim de otimizar as condições reacionais. Os resultados podem ser visualizados na Tabela .5 . 
Research, Society and Development, v. 11, n. 2, e25011225652, 2022

(CC BY 4.0) | ISSN 2525-3409 | DOI: http://dx.doi.org/10.33448/rsd-v11i2.25652

Tabela 5: Resultados de conversão e seletividade para as reações com o catalisador $\mathrm{CuSiO}_{2}-\mathrm{D} 2$.

\begin{tabular}{cccc}
\hline Reação & Conv. (\%) & Sel. (\%) & Rendimento (\%) \\
\hline 1 & 12,38 & 75,77 & 9,38 \\
2 & 32,81 & 6,61 & 2,17 \\
3 & 28,17 & 94,14 & 26,52 \\
4 & 47,20 & 48,20 & 22,75 \\
5 & 3,35 & 13,43 & 0,45 \\
6 & 3,00 & 33,33 & 1,00 \\
7 & 24,24 & 90,14 & 21,85 \\
8 & 25,54 & 95,02 & 24,27 \\
9 & 59,95 & 69,42 & 40,92 \\
\hline
\end{tabular}

Fonte: Autores.

Figura 5: A) Conversão de glicerol e B) Seletividade a ácido lático em diferentes concentrações de glicerol (0,6 M e 3,3 M) e diferentes temperaturas reacionais $\left(220,250,280,290\right.$ e $\left.300^{\circ} \mathrm{C}\right)$. Condições reacionais: razão mássica $\mathrm{NaOH} / \mathrm{glicerol}=1: 1$ e $0.75 \mathrm{~g}$ de catalisador $\mathrm{Cu} / \mathrm{SiO}_{2}-\mathrm{D} 2$.

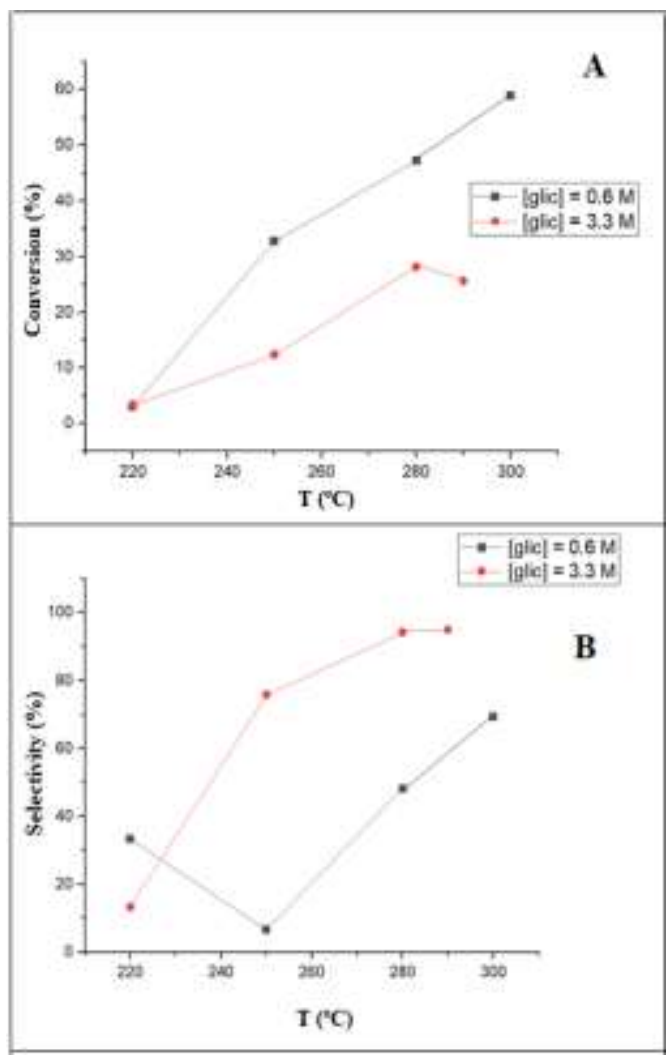

Fonte: Autores.

Ao analisar os resultados da Tabela 5 e da Figura 5, é possível observar que uma menor concentração de glicerol possui maior efeito positivo no parâmetro de conversão quando comparado a uma maior concentração de glicerol. Sendo assim, os resultados indicam uma tendência de crescimento da conversão à medida que se aumenta a temperatura reacional, corroborando com o descrito por Kishida et al. (2005). Entretanto, essa evidência fica mais expressiva quando se utilizam menores concentrações de glicerol, como é o caso da concentração de 0,6M. Resultados semelhantes já foram apontados por 
Ramírez-López et al. (2010). que notaram queda expressiva na conversão de glicerol à medida que se aumentou a concentração de glicerol na reação.

Observa-se que uma maior concentração de glicerol $(3,3 \mathrm{M})$ levou à maior seletividade a ácido lático quando comparado à menor concentração $(0,6 \mathrm{M})$. Entretanto, para analisar este parâmetro de seletividade é necessário aliar ao parâmetro conversão de glicerol, de maneira que ambos apresentem bons resultados. Ao atrelar os parâmetros conversão de glicerol e seletividade, observa-se que a conversão de glicerol é bem mais baixa quando se utiliza uma maior concentração de glicerol. Isso pode ser explicado pela possibilidade de também ocorrer conversão de glicerol a outros produtos gasosos, desfavorecendo a conversão de glicerol a ácido lático quando houver aumento da concentração do mesmo no meio reacional, conforme descrito por Kishida et al. (2005).

De acordo com os resultados anteriores, observou-se que as melhores condições para o processo de conversão hidrotérmica alcalina do glicerol com este catalisador $\left(\mathrm{Cu} / \mathrm{SiO}_{2}\right.$ - $\left.\mathrm{D} 2\right)$ são uma temperatura reacional de $300^{\circ} \mathrm{C}$, uma razão molar $\mathrm{NaOH} /$ glicerol igual a 1, uma concentração igual a 0,6M para o glicerol e o hidróxido de sódio e um tempo reacional de $6 \mathrm{~h}$, obtendo os maiores resultados de conversão $(58,95 \%)$, seletividade a ácido lático $(69,42 \%)$ e rendimento de ácido lático $(40,92 \%)$.

Como a tendência de conversão e seletividade a ácido lático diferem em relação à concentração do glicerol, o rendimento de ácido lático foi baixo para os experimentos realizados, apresentando o melhor resultado de rendimento $(40,92 \%)$ para as melhores condições reacionais citadas anteriormente, motivo que justificou a utilização desta concentração de $0,6 \mathrm{M}$ de glicerol para os posteriores experimentos.

\subsubsection{Influência da Temperatura}

Para o catalisador $\mathrm{Cu} / \mathrm{SiO}_{2}-\mathrm{F} 1$, foi avaliada a influência da temperatura de otimizar as condições reacionais. Os resultados podem ser observados na Tabela 6.

Tabela 6: Conversão de glicerol e seletividade a ácido lático com catalisador $\mathrm{Cu} / \mathrm{SiO}_{2}-\mathrm{F} 1$.

\begin{tabular}{ccccccc}
\hline Reação & {$[\mathbf{N a O H}]\left(\mathbf{g ~ L}^{-\mathbf{1}}\right)$} & {$[\mathrm{glic}]\left(\mathbf{g ~ L}^{\mathbf{1}}\right)$} & $\mathbf{t}(\mathbf{h})$ & $\mathbf{T}\left({ }^{\circ} \mathbf{C}\right)$ & $\begin{array}{c}\text { Conv. } \\
(\%)\end{array}$ & Sel. (\%) \\
\hline 10 & 0,6 & 0,6 & 6 & 250 & 35,44 & 39,51 \\
11 & 0,6 & 0,6 & 6 & 280 & 44,44 & 45,66 \\
12 & 0,6 & 0,6 & 6 & 300 & 53,98 & 59,76 \\
\hline
\end{tabular}

Fonte: Autores. 
Figura 6: A) Conversão de glicerol e B) Seletividade a ácido lático em diferentes concentrações de glicerol (0.6M e 3.3M) e diferentes temperaturas reacionais $\left(220,250,280,290\right.$ e $\left.300^{\circ} \mathrm{C}\right)$. Condições reacionais: razão mássica $\mathrm{NaOH} / \mathrm{glicerol}=1: 1$ e $0.75 \mathrm{~g}$ de catalisador $\mathrm{Cu} / \mathrm{SiO}_{2}-\mathrm{D} 2$.

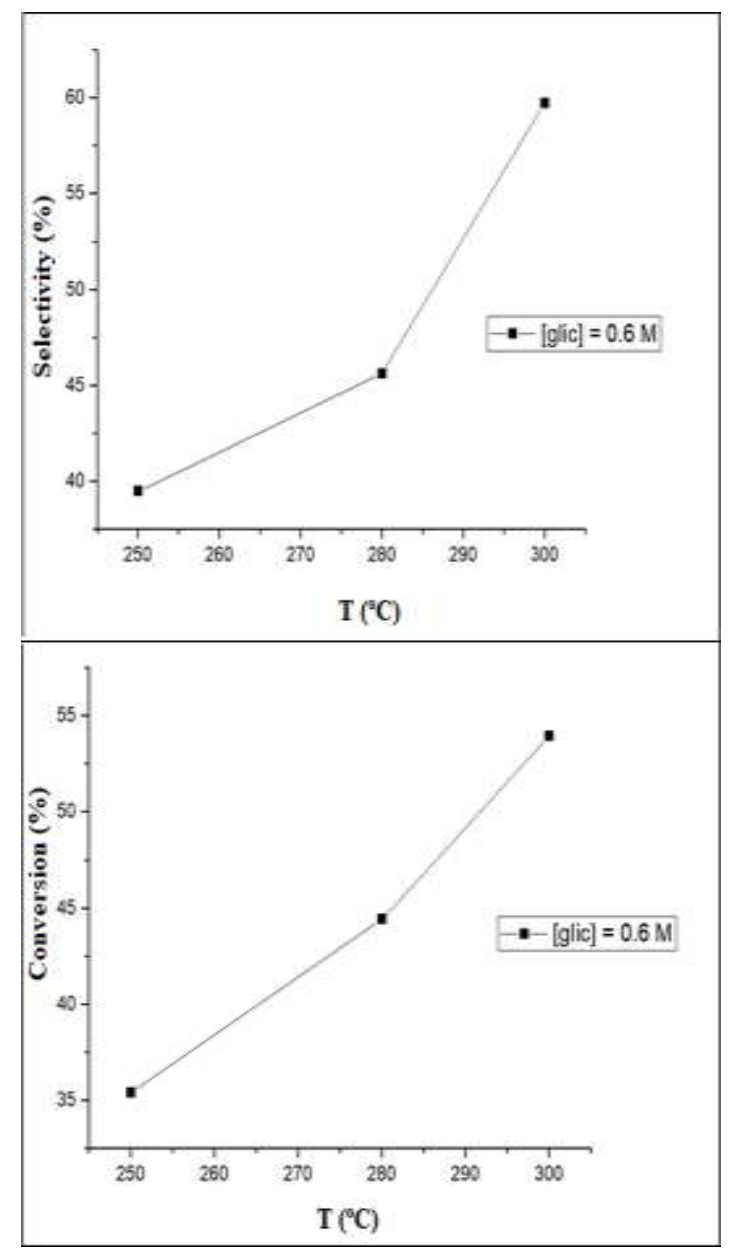

Fonte: Autores.

Através da Tabela 6 e Figura 6, observa-se que a seletividade ao ácido lático foi maior para o processo catalítico a temperatura de $300^{\circ} \mathrm{C}$, apresentando um aumento progressivo à medida que o tempo de reação aumenta, mostrando que esses parâmetros possuem uma relação matemática diretamente proporcional, o que é um descrito por Evans et al. (2020).

Foi possível observar que as melhores condições para o processo de conversão hidrotérmica alcalina do glicerol com este catalisador $\left(\mathrm{Cu} / \mathrm{SiO}_{2}-\mathrm{F} 1\right)$ são uma temperatura de reação de $300^{\circ} \mathrm{C}$, uma relação mássica $\mathrm{NaOH} / \mathrm{glicerol}$ igual a 1 , uma concentração igual a 0,6M para glicerol e hidróxido de sódio e tempo de reação de 6h, atingindo os maiores resultados de conversão $(53,98 \%)$ e seletividade ao ácido lático $(59,76 \%)$.

\section{Conclusão}

As análises realizadas foram eficientes para a caracterização de catalisadores de cobre suportados em sílica. Através da análise das propriedades texturais, foi possível classificar os diâmetros médios dos poros dentro da faixa de mesoporos, de acordo com a IUPAC. Ao comparar os três catalisadores, observou-se que o catalisador $9 \% \mathrm{Cu} / \mathrm{SiO}_{2}-\mathrm{F} 1$ apresentou o maior diâmetro volumétrico, enquanto o $9 \% \mathrm{Cu} / \mathrm{SiO}_{2}-\mathrm{D} 2$ apresentou o menor diâmetro volumétrico.

Comparando os valores da área superficial do suporte com os catalisadores suportados em $\mathrm{SiO}_{2}$, observou-se que a redução mais significativa na área superficial ocorreu para o catalisador $9 \% \mathrm{Cu} / \mathrm{SiO}_{2}-\mathrm{D} 2$ e a menor para o catalisador 
9\% $\mathrm{Cu} / \mathrm{SiO}_{2}-\mathrm{F} 1$, fato que pode ser explicado pela distribuição de poros no suporte de sílica e também bloqueio por partículas metálicas.

A análise de FRX indicou uma síntese eficiente dos catalisadores uma vez que as diferenças entre as quantidades teoricamente previstas para a composição dos catalisadores $(\mathrm{Cu}, \mathrm{Mg}$ e $\mathrm{Si})$ e as quantidades reais obtidas na análise ocorrem conforme o esperado. Assim, conclui-se que o método utilizado para a síntese dos catalisadores foi adequado.

A difração de raios $\mathrm{X}$ foi eficiente para identificar as fases cristalinas formadas. Observou-se que todos os catalisadores de cobre apresentaram perfil semelhante, notando-se diferenças apenas quanto à intensidade do pico. Concluiu-se que o grande tamanho do cristal proporciona boa estabilidade e melhor contato entre as partículas de cobre e sílica, proporcionando um melhor desempenho dos catalisadores $\mathrm{Cu} / \mathrm{SiO}_{2}$.

As análises de TPR- $\mathrm{H}_{2}$ mostraram que todos os catalisadores de cobre iniciaram sua redução com uma temperatura na faixa de 240 a $260^{\circ} \mathrm{C}$, fato justificável pela igual proporção de catalisador utilizado. Os perfis de TPR indicaram que a redução de $\mathrm{CuO}$ ocorreu em temperaturas abaixo de $600 \mathrm{~K}$ em todos os catalisadores, valores dentro da faixa esperada na literatura.

As análises de TPD- $\mathrm{NH}_{3}$ foram adequadas para determinar a acidez dos catalisadores, sendo possível observar que a combinação de sítios ácidos fracos e fortes presente nos catalisadores foi benéfica para o desempenho catalítico, influenciando positivamente na conversão do glicerol e na seletividade do ácido lático.

Ao analisar o desempenho catalítico das reações realizadas, no caso do catalisador $\mathrm{Cu} / \mathrm{SiO}_{2}-\mathrm{D} 2$, estudou-se a influência do parâmetro de concentração de glicerol nas reações, observando que uma concentração mais baixa de glicerol leva a um efeito positivo mais significativo no parâmetro de conversão. Os resultados indicam um aumento na conversão à medida que a temperatura da reação aumenta, corroborando com a literatura. Ao correlacionar os parâmetros de conversão e seletividade, os melhores resultados são observados quando se utilizam concentrações mais baixas de glicerol $(0,6 \mathrm{M})$. O aumento na concentração pode levar a um aumento nos subprodutos e a uma diminuição na seletividade do ácido lático. Assim, as melhores condições para este catalisador $\left(\mathrm{Cu} / \mathrm{SiO}_{2}-\mathrm{D} 2\right)$ foram temperatura de reação de $300^{\circ} \mathrm{C}$, razão molar $\mathrm{NaOH} /$ glicerol $=1: 1$, concentrações de glicerol e hidróxido de sódio iguais a $0,6 \mathrm{M}$ e tempo de reação de $6 \mathrm{~h}$, obtendo resultados conversão satisfatória $(58,95 \%)$ e seletividade ao ácido lático $(69,42 \%)$.

Para o catalisador $\mathrm{Cu} / \mathrm{SiO}_{2}$-F1, avaliou-se a influência do parâmetro de temperatura de reação nas reações realizadas. Um aumento progressivo na conversão do glicerol e na seletividade ao ácido lático foi observado à medida que a temperatura da reação aumentou, em linha com o que é esperado na literatura. Portanto, as melhores condições para este catalisador são uma temperatura de reação de $300^{\circ} \mathrm{C}$, uma relação mássica de $\mathrm{NaOH} /$ glicerol igual a 1:1, concentrações de glicerol e hidróxido de sódio iguais a $0,6 \mathrm{M}$ e um tempo de reação de $6 \mathrm{~h}$, alcançando os maiores resultados de conversão $(53,98 \%)$ e seletividade ao ácido lático $(59,76 \%)$.

Como sugestões futuras deste trabalho estão realizar um planejamento experimental para avaliar a influência das variáveis dependentes na otimização das reações de conversão do glicerol a ácido lático; testar outros suportes para os catalisadores, além da sílica $\left(\mathrm{SiO}_{2}\right)$ já utilizada; testar e comparar outras bases nestas reações; avaliar as reações em tempos maiores do que 6 horas, verificando a vida útil do catalisador; avaliar a reutilização dos catalisadores após regeneração; realizar as mesmas reações de conversão hidrotérmica do glicerol em escala industrial.

\section{Agradecimentos}

Ao CNPq pela bolsa concedida durante a execução desta pesquisa. 


\section{Referências}

Adhikari, S., Fernando, S. D. \& Harvanto, A. S. D. (2008). Hydrogen production from glycerin by steam reforming over nickel catalysts, Renew. Energy, 33, 1097-1100.

Dussault, L., Dupin, J. C., Dumitriu, E., Auroux, A. \& Guimon, C. (2005). Microcalorimetry, TPR and XPS studies of acid-base properties of NiCuMgAl mixed oxides using LDHs as precursors, Thermochimica Acta, 434, 93-99.

Evans, M. D., Douthwaite, M., Carter, J. H., Pattisson, S., Kondrat, S. A., Bethell, D., Knight, D. W., Taylor, S. H. \& Hutchings, G. J. (2020). Enhancing the understanding of the glycerol to lactic acid reaction mechanism over AuPt/TiO ${ }_{2}$ under alkaline conditions. The Journal of Chemical Physics, 152, 534-542.

Flores, J. H., Peixoto, D. P. B., Appel, L. G. \& Avillez, R. R. (2011). The influence of different methanol synthesis catalysts on direct synthesis of DMF from syngas. Catalysis Today, 172, 218-225.

Huang, Z., Cui, F., Xue, J., Zuo, J., Chen, J. \& Xia, C. (2012). Cu/SiO 2 catalysts prepared by hom- and heterogeneous deposition-precipitation methods: texture, structure and catalytic performance in the hydrogenolysis of glycerol to 1,2-propanediol. Catalysis Today, $183,42-51$.

Huynh, T.M., Armbruster, U., Kreyenschulte, C. R., Nguyen, L. H., Phan, B. M. Q., Nguyen, D. A. \& Martin, A. (2016). Understanding the performance and stability of supported Ni-Co-based catalysts in phenol HDO. Catalysts, 6, 176-196.

Jiao, W.Q., Yue, M. B., Wang, Y. M. \& He, M. Y. (2012). Synthesis of morphology-controlled mesoporous transition aluminas derived from the decomposition of alumina hydrates. Microporous and Mesoporous Materials, 147 (1), 167-177.

Karge, H. G., Dondur, V. \& Weitkamp, J. (1991). Investigation of the distribution of acidity strength in zeolites by temperature-programmed desorption of probe molecules. 2. Dealuminated Y-type zeolites. The Journal of Physical Chemistry, 95 (1), 283-288.

Kishida, H, Fangming, J., Zhouyu, Z., Takehiko, M. \& Heiji, E. (2005). Conversion of Glycerin into Lactic Acid by Alkaline Hydrothermal Reaction. Chemistry Letters, 34 (11),1560-1561.

Liu, X., Lang, W. Z., Long, L. \& Hu, C.L. (2014). Improved catalytic performance in propane dehydrogenation of $\mathrm{PtSn} / \gamma_{-} \mathrm{Al}_{2} \mathrm{O}_{3}$ catalysts by doping indium. Chemical Engineering Journal, 247, 183-192.

Maki-Arvela, P., Simakova, I. L., Salmi, T. \& Murzin, D. Y. (2014). Production of Lactic acid/Lactates from Biomass and their catalytic transformations to commodities. Chemical Reviews, 114 (3), 1909-1971.

Marconi, M. A., \& Lakatos, E. M. (2017). Fundamentos de metodologia científica (8a ed.). São Paulo: Atlas.

Postaue, N., Trentini, C. P. \& Silva, C. S. (2019). Produção de biodiesel com metilacetato pressurizado sem geração de glicerol. Revista UNINGÁ Review, 34 (3), 14-28.

Ramírez-López, C. A., Ochoa-Gómez, J. R., Fernández-Santos, M., Gómez-Jiménez-Aberasturi, O., Alonso-Vicario, A. \& Torrecilla-Soria, J. (2010). Synthesis of lactic acid by alkaline hydrothermal conversion of glycerol at high glycerol concentration. Industrial and Engineering Chemistry Research, 49 (14), 6270-6278.

Roy, D., Subramaniam, B. \& Chaudhari, R. V. (2011). Cu-based catalysts show low-temperature activity for glycerol conversion to lactic acid. ACS Catalysis, $1,548-551$

Shahbaz, M., Raghutla, C., Chittedi, K. R., Jiao, Z. \& Vo, X. V. (2020). The effect of renewable energy consumption on economic growth: evidence from the renewable energy country attractive index. Energy, 207, 1-14.

Sing, K. S. W. (1985). Reporting physisorption data for gas/solid systems with particular reference to determining surface área and porosity. Pure \& Applied Chemistry, 57 (4), 603-619.

Tran, N. T.T., Uemura, Y., Chowdhury, S. \& Ramli, A. (2016). Vapor-phase hydrodeoxygenation of guaiacol on Al-MCM-41 supported Ni and Co catalysts. Applied Catalysis A: General, 512, 93-100.

Wang, L., Gaudet, J. R., Li, W., Weng, D. (2013). Migration of Cu species in Cu/SAPO-34 during hydrothermal ageing. Journal of Catalysis, $306,68-77$.

Webb, P. A. \& Orr, C. (1997). Analytical Methods in Fine Particle Technology. Micromeritics Instrument.

Yin, H., Zhang, C., Yin, H., Gao, D., Shen, L. \& Wang, A. (2017). Hydrothermal conversion of glycerol to lactic acid catalyzed by Cu/hydroxyapatite, $\mathrm{Cu} / \mathrm{MgO}$, and $\mathrm{Cu} / \mathrm{ZrO}_{2}$ and reaction kinetics. Chemical Engineering Journal, 288, 332-343. 